\title{
Uso de preprints en congresos científicos como alternativa a la publicación de actas: la experiencia del IX Encuentro Ibérico EDICIC 2019
}

\author{
Use of preprints in scientific conferences as an alternative to the publication of \\ proceedings: the experience of the IX Encuentro Ibérico EDICIC 2019
}

Preprint: v1.0 (12/01/2021), bajo licencia internacional CC BY 4.0

\author{
Cristóbal Urbanoํㅡㄴ (https://orcid.org/0000-0003-0935-6436) [urbano@ub.edu] \\ Sara Tafalla ${ }^{2}$ \\ Ángel Borrego ${ }^{1}$ (https://orcid.org/0000-0002-6462-3966) \\ Ernest Abadal ${ }^{1}$ (https://orcid.org/0000-0002-9151-6437) \\ ${ }^{1}$ Universitat de Barcelona, Departament de Biblioteconomia, Documentació i Comunicació Audiovisual \& \\ Centre de Recerca en Informació, Comunicació i Cultura. ${ }^{2}$ Comité Organizador EDICIC 2019 Barcelona, \\ Secretaria Técnica.
}

Resumen: Los preprints están adquiriendo cada vez mayor relevancia como canal para la comunicación rápida de los resultados de la investigación. Este trabajo analiza su uso en el congreso EDICIC 2019, en el que se solicitó a los autores de comunicaciones el depósito de sus preprints antes del encuentro con el objeto de impulsar una mayor interacción entre los asistentes y potenciar la posterior publicación de los trabajos en revistas arbitradas como sustituto de las actas. Los resultados de una encuesta entre los autores de correspondencia de las comunicaciones presentadas muestran una escasa familiaridad con este formato. No obstante, casi tres cuartas partes de los autores depositaron el preprint de su comunicación antes del inicio del encuentro. La mayoría de los encuestados está de acuerdo en que un congreso funciona mejor cuando los preprints han estado previamente a disposición de los asistentes y son favorables a limitar el tiempo de presentación por parte de los autores para dejar más espacio al debate con los asistentes. En menor medida, los encuestados verían con buenos ojos que este modelo se aplicara en otros congresos de Información y Documentación, aunque hay discrepancias sobre si las revistas del área están preparadas para la aceptación de los preprints en el marco de los principios de la ciencia abierta. Los autores que habían depositado su comunicación como preprint antes del congreso fueron más proclives a enviar posteriormente su trabajo a una revista para su publicación formal como artículo. Finalmente, los encuestados mostraron su preocupación por la falta de reconocimiento de su participación en el congreso al no publicarse las actas; destacaron el doble esfuerzo que supone superar la evaluación de los organizadores del congreso y, posteriormente, de la revista; recalcaron que la cultura de los preprints y de la ciencia abierta todavía no está consolidada entre las revistas de Información y Documentación; y constataron que el incremento de la interacción y el debate en las sesiones de comunicaciones, objetivo último que se pretendía conseguir con la difusión anticipada de las comunicaciones, no se alcanzó plenamente.

Palabras clave: ciencia abierta; comunicación científica; congresos; preprints; revistas científicas, actas de congreso 


\begin{abstract}
Preprints are increasingly relevant for fast dissemination of research results. This article analyses its use in the EDICIC 2019 conference, where authors were requested to deposit their preprints before the conference in order to encourage interaction among participants and to push their publication in refereed journals as a substitute for conference proceedings. The results of a survey among corresponding authors of accepted papers show little familiarity with preprints. Nevertheless, nearly three-quarters of authors deposited their preprint before the conference. Most of them agree that a conference runs more smoothly if preprints are available to attendees before the event and agree to reduce time slots devoted to presentations in order to encourage the debate between presenters and attendees. To a lesser extent, survey respondents would like to see this model implemented in other Library and Information Science conferences, although they disagree on whether journals from that area are ready to accept preprints in the framework of open science principles. Authors who had deposited their preprint before the conference were more willing to submit their work to a journal for formal publication as an article. Finally, surveyed authors expressed their concerns regarding the lack of recognition of their participation if conference proceedings are not published; highlighted the effort made to pass a double evaluation by conference organisers and journal editors; emphasized that the culture of preprints and open science is not consolidated among disciplinary journals as yet; and noted that the aim of increasing interaction and debate was not fully reached.
\end{abstract}

Keywords: conferences; open science; preprints; scholarly communication; scholarly journals; conference proceedings

\title{
1 Introducción
}

Los manuscritos de artículos científicos pueden tardar más de un año en publicarse en revistas reconocidas ya que deben superar un lento proceso de recepción, revisión por expertos, edición, maquetación y publicación final. Esto supone una importante limitación en el funcionamiento del sistema de comunicación científica dado que los progresos de la investigación no pueden ser compartidos con la celeridad adecuada. Para solucionar este problema, desde la década de 1990 han proliferado los repositorios de preprints, que ponen estos manuscritos a disposición de la comunidad científica de manera inmediata en acceso abierto. Su crecimiento ha sido notable en los últimos años con el impulso del modelo de ciencia abierta, y particularmente desde inicios de 2020 con la intensificación de la investigación sobre la Covid-19. Sin embargo, su aceptación y uso es muy desigual según las diversas áreas de conocimiento, al tiempo que el debate de la fiabilidad de los contenidos de los repositorios se ha vuelto cada vez más intenso a medida que el modelo de preprints se extiende a nuevas áreas.

Ahora bien, mucho antes de la expansión de los repositorios de preprints, los congresos y todo tipo de reuniones científicas ya jugaban un papel importante en la comunicación ágil 
de resultados de investigaciones en curso o ya finalizadas, en ocasiones en forma preliminar. En función de la tradición y la naturaleza particular de cada evento, este tipo de encuentros permiten a ciertos investigadores recibir retroalimentación de los colegas para perfeccionar sus trabajos, así como obtener el reconocimiento y registro de los mismos mediante la publicación formal de proceedings. Sin embargo, con excepción de algunas áreas de conocimiento muy específicas, el atractivo que para muchos autores tiene hoy en día presentar comunicaciones a congresos viene siendo decreciente, tanto por los costes que implican los desplazamientos e inscripciones, como por el bajo reconocimiento que tiene este tipo de contribuciones, en comparación con los artículos en revistas arbitradas, en los criterios usados por un buen número de agencias de evaluación y acreditación. Junto a ese problema de falta de retorno en forma de prestigio para el currículum de los investigadores, también son diversas las voces que constatan un cierto agotamiento de las fórmulas más tradicionales utilizadas en la organización de reuniones científicas presenciales, ya que el grado de participación e interacción entre los asistentes con frecuencia no es plenamente satisfactorio.

En ese contexto y como una contribución al debate sobre la posible conexión entre preprints y congresos, este trabajo presenta la evaluación del uso de preprints durante el IX Encuentro Ibérico de la Asociación de Educación e Investigación en Ciencia de la Información de Iberoamérica y el Caribe (EDICIC) [https://fima.ub.edu/edicic2019/], entidad que congrega instituciones y personas del área de educación e investigación en Ciencia de la Información (Bibliotecología, Documentación y Archivística) de Iberoamérica y el Caribe.

Con el objetivo de impulsar una mayor interacción entre los asistentes y potenciar la posterior publicación de los trabajos en revistas arbitradas, se renunció a la publicación formal de proceedings. Así, una vez evaluados los resúmenes de las propuestas de comunicación por doble ciego, se instó a los autores a subir como preprint al repositorio de su elección el texto de las comunicaciones aceptadas, y se difundieron en abierto antes de la celebración del encuentro mediante el catálogo de comunicaciones [https://fima.ub.edu/edicic2019/catalogo]. Dado que muchas revistas iberoamericanas del área de Información y Documentación no establecían claramente la aceptación o rechazo de originales procedentes de preprints, se realizaron gestiones para dar seguridad a los autores mediante una lista de revistas que respondieron positivamente la consulta de los organizadores sobre si aceptarían preprints generados por el IX Encuentro Ibérico EDICIC. También se ofreció en el web del Encuentro orientación sobre la naturaleza de los preprints y se organizó un webinar sobre la cuestión (Seminario CRICC, 2019).

El Encuentro recibió 225 propuestas de comunicación y 21 de pósteres. Una vez evaluados los resúmenes, se materializaron en forma de presentación final efectiva 113 comunicaciones en las que se identificaron 292 autorías y 58 pósteres con 155 autorías, 
pese a que el número total de autores únicos fue menor pues algunos participaron en más de una comunicación o póster. El total de asistentes fue de 372 , entre los que se contabilizaron al menos un autor por cada contribución, miembros del comité científico y organizador, 40 participantes que presentaron su proyecto de tesis en el consorcio doctoral asociado al evento, así como otros asistentes sin contribución formal. Si nos fijamos en los autores de las comunicaciones sobre los cuales centraremos nuestro estudio, cabe señalar que en las 113 comunicaciones presentadas se identificaron un total de 292 autorías, de las que casi dos tercios $(188,64 \%)$ procedían de Brasil, 58 de España (20\%) y 38 de Portugal (13\%). Las 8 autorías restantes (3\%) procedieron de Argentina, Canadá, Italia, Países Bajos, Puerto Rico e Italia.

El modelo de presentación de comunicaciones basado en preprints rompía con una tradición consolidada de publicación formal de las actas de los encuentros del Capítulo Ibérico de EDICIC. Supuso un riesgo, ya que una buena parte de los asistentes tradicionalmente han contado con esa publicación formal como aportación a su currículum, algo que en el modelo planteado se posponía al posterior envío del preprint a una revista, pero sin seguridad de publicación pese a que la comunicación hubiera sido aceptada para su presentación oral. Por ello, el comité organizador local acordó con el equipo directivo del Capítulo Ibérico de EDICIC el carácter experimental de la edición 2019 y la necesidad de realizar el presente estudio para evaluar los resultados obtenidos y la opinión de los autores de las comunicaciones. Dicho estudio se sustenta en las siguientes preguntas de investigación:

1. ¿Qué experiencia previa del uso de preprints tenían los autores que presentaron comunicaciones a EDICIC 2019?

2. ¿En qué medida dichos autores se involucraron en el modelo de presentación de comunicaciones mediante preprints propuesto por la organización de EDICIC 2019?

3. ¿Cómo afectó el modelo de comunicaciones en forma de preprints la participación de los asistentes en las sesiones públicas de presentación de comunicaciones?

4. ¿En qué grado las comunicaciones han sido posteriormente objeto de publicación formal en revistas?

La respuesta a dichas preguntas ofrece la oportunidad de enriquecer el debate sobre el papel actual de los congresos en la comunicación académica y sobre la necesidad de innovar en los formatos de congreso. Si bien este estudio se realizó con el propósito de complementar el proceso de evaluación del evento, el impacto que ha tenido la pandemia de Covid-19 en infinidad de congresos de todo tipo, que han experimentado la suspensión o su transformación digital (Clarke, 2020), pone de relieve el interés de este estudio. Una seria reflexión sobre la razón de ser de los congresos presenciales y, de forma particular, sobre el futuro de las actas de congreso como formato de publicación científica, se contempla como inevitable. 


\section{Preprints: definición, evolución y prospectiva}

Se consideran preprints aquellos documentos científicos que no han pasado aún el proceso de peer review y que se comparten en repositorios para ofrecer los resultados de una investigación de manera inmediata. Diversos autores han revisado las diversas definiciones de preprint (p.e. Balaji \& Dhanamjaya, 2019; Tennant, Bauin et al., 2018), mostrando los diversos matices de un término que pese su vínculo con el mundo digital sigue remitiendo en su etimología al mundo de la edición analógica. En todo caso, lo distintivo del término remite a la etapa de la comunicación científica en la que se ubica, tal y como queda claramente expuesto en la definición que ofrece el editor PLOS en la sección sobre "Open Science" de su sitio web: "A preprint is a version of a scientific manuscript posted on a public server prior to formal peer review" (PLOS, 2021).

Como vemos, la característica fundamental y distintiva de un preprint es no haber pasado por el peer review. Parecería, por tanto, que lo opuesto sería el postprint, es decir, la versión revisada y publicada. De todas formas, Eysenbach (2000) señala que el término puede llevar a una cierta confusión, ya que la palabra preprint parece indicar que se trata de documentos que acabarán siendo publicados formalmente, lo cual no es cierto dado que los documentos que están alojados en repositorios de preprints pueden no ser enviados nunca a una revista, o no ser aceptados por ellas en caso de envío. Para clarificar las cosas y no limitarse a la oposición preprint/postprint, algunos autores (Tennant, Bauin et al., 2018) recomiendan tener en cuenta la terminología del directorio Sherpa-Romeo [https://v2.sherpa.ac.uk/romeo/] para los diversos estados de un documento:

1. Versión del autor, equivalente a Preprint [= Submitted Version, Original Manuscript];

2. Versión aceptada, equivalente a Postprint [= Authors Accepted Manuscript (AAM), Final Author Version], correspondiente al texto final tal y como resulta de la revisión del manuscrito aceptada por una revista, pero sin maquetar;

3. Versión publicada [= Version of Record], correspondiente a la versión exacta maquetada que publica la revista.

Atendiendo al origen histórico de esta tipología documental, es importante recordar que la circulación de manuscritos no publicados es anterior a la misma existencia de las revistas científicas, como prueba el estudio de la correspondencia manuscrita entre científicos, especialmente intensa a partir de la llustración. Por otra parte, a lo largo de la historia han sido muchos los congresos que antes o durante la celebración del evento han ayudado a la circulación del contenido de dichos manuscritos no publicados formalmente. Además, durante buena parte del periodo "pre-Internet" del siglo XX fueron numerosos los proyectos de distribución analógica de preprints, como por ejemplo fue el caso entre 1961 y 1966 de los "Information Exchange Groups" de los National Institutes of Health 
(NIH) de EEUU (Confrey, 1966). Sin embargo, lo que define en la actualidad al preprint es su archivo público en un repositorio digital. El punto de arranque de ese concepto se asocia al repositorio arXiv.org [https://arxiv.org/], creado en 1991 para el ámbito de la física, las ciencias de la computación y las matemáticas, que es reconocido como el repositorio paradigmático de preprints que abrió el camino al modelo actual (Ginsparg, 2011). La rápida aceptación de arXiv.org ayudó a que ese modelo se extendiese a otras disciplinas durante la década de 1990, con ejemplos como Social Sciences Research Network (SSRN) en 1994 [https://www.ssrn.com] y Research Papers in Economics (RePEc) en 1997 [http://www.repec.org/], aunque hay que tener presente que estos repositorios contienen también otros documentos que no son preprints. Más recientemente, en la década de 2010, se ha dado un nuevo y más amplio impulso con ejemplos como bioRxiv en 2013 [https://www.biorxiv.org/], socArXiv en 2016 [https://osf.io/preprints/socarxiv/] o medRxiv en 2019 [https://www.medrxiv.org/], por citar tan sólo algunos de los muchos ejemplos que podríamos encontrar.

Existe una tipología variada de repositorios que a veces es difícil de comparar entre sí. Rodríguez (2019) establece una categorización que puede ser útil para clarificar el panorama: servidores originales especializados en preprints (p.e. arXiv.org), servidores vinculados a revistas (p.e. Advance de Sage), repositorios temáticos (p.e. E-LIS), repositorios estatales (p.e. Chinese Preprint Server), y servicios con funcionalidades diversas (p.e. GitHub o ResearchGate). Por su parte, Balaji \& Dhanamjaya (2019) analizan y clasifican los repositorios de preprints según sus características técnicas, la arquitectura, la interoperabilidad, las métricas, el software utilizado, etc.

Como se ha señalado, algunos editores y portales de revistas también han impulsado sus propios repositorios. Sería el caso de Preprints [https://www.preprints.org/] creado en 2016 por MDPI para dar este servicio a los manuscritos enviados a sus centenares de revistas, o de SciELO-Preprints [https://preprints.scielo.org/] que tras años de análisis (Packer et al, 2017) ha entrado en funcionamiento en 2020 sobre la plataforma Open Preprint Systems desarrollada conjuntamente con PKP [https://pkp.sfu.ca/ops/], en una apuesta por introducir este modelo entre las revistas de acceso abierto de orientación nocomercial. Por su parte, PLOS ofrece esta funcionalidad desde 2018 bien en colaboración directa con bioRxiv y medRxiv (que se manifiesta en una pasarela de conexión entre esos repositorios y su sistema de gestión de envío de manuscritos), bien animando a los autores a enviar los manuscritos al repositorio de su respectiva disciplina antes o al tiempo que se postulan ante las revistas. Una estrategia similar a la adoptada cada vez más por revistas de todo tipo y modelo de negocio.

$Y$ es que, hoy por hoy, los preprints no se entienden como una alternativa completa a las revistas, sino como una etapa previa. De hecho, las revistas tienen un papel importante en el crecimiento y en la introducción de mejoras técnicas para la gestión de los preprints. En 
primer lugar, no tan solo aceptando la publicación de artículos difundidos previamente como preprints sino facilitando y promoviendo esta posibilidad. En segundo lugar, procurando mantener la conexión (vía identificadores persistentes) entre la versión del autor (preprint) y la versión publicada. Esta cuestión debe ser también seguida por parte de los repositorios de preprints, que deberían estimular el registro de los cambios de estado de los manuscritos, facilitando información actualizada de las diversas versiones (Neylon et al., 2017). En un estudio sobre políticas editoriales respecto de los preprints (Teixeira da Silva \& Dobranzki, 2019) quedaba claro que cada vez más revistas aceptan e incluso estimulan los preprints: el $78 \%$ de las 14 grandes editoriales científicas analizadas tenían política de preprints en 2018 y, además, en la mayoría de los casos, se pedía al autor información sobre la existencia del preprint.

Sin duda, la creciente aceptación y uso de los preprints, así como la ampliación del alcance disciplinar que se observa en la aparición de nuevos repositorios, parecen indicar un saldo positivo en el balance entre ventajas e inconvenientes de una incorporación regular de los preprints al ciclo de la comunicación científica. El análisis de dicho balance ha sido comentado por diversos autores (p.e. Berg et al., 2016; Bourne et al., 2017; Elmore, 2018; Fry et al., 2019; Neylon et al., 2017; Ng, 2017) a partir de los cuales podemos destacar, entre otros, los siguientes puntos fuertes:

1. La comunicación inmediata de los contenidos mediante preprints permite acelerar el progreso de la investigación y de la ciencia en general, al tiempo que permite anticipar el registro público de la autoría.

2. La ampliación del número de expertos a los que se exponen los trabajos para su revisión abierta. Ello da pie a recibir muchos puntos de vista y comentarios antes de enviar los manuscritos a las revistas, cuyos sistemas de evaluación de originales se suelen limitar a dos o tres revisores.

3. La difusión de contenidos más innovadores. Los preprints se pueden usar para exponer ideas o investigaciones poco adecuadas para su publicación en medios convencionales.

4. El incremento de la visibilidad y del impacto de los trabajos, así como una mejor detección temprana del interés que generan los resultados de las investigaciones recientes. Esas ventajas en términos de impacto han sido objeto de estudios que en general confirman que los trabajos publicados en revistas procedentes de preprints reciben un mayor número de citas (Brown, 2001 y 2003; Feldman et al., 2018; Fu y Hughey, 2019; Henneken et al., 2006; Wang, Chen et al., 2020; Wang, Glänzel et al. 2020).

Esos puntos fuertes están impulsando la penetración del sistema de preprints, pero no hay que olvidar que existen diversos puntos débiles que se mencionan en la bibliografía, aunque con una afectación diversa en función de los ámbitos disciplinares y sus diferentes 
culturas de comunicación y debate científico. Podríamos decir que los problemas se centran en el riesgo de proliferación de trabajos poco originales, metodológicamente débiles, o con datos y conclusiones incorrectos, que pueden generar inflación en el sistema de comunicación, así como desinformación y falsas expectativas entre públicos poco especializados, o sin suficiente capacidad crítica. Este aspecto es especialmente delicado cuando es la prensa la que proyecta información de los preprints a la sociedad en general (Sheldon, 2018; Tennant, Gatto et al., 2018).

Ahora bien, los problemas de fiabilidad y de inflación de información, que se apuntan como los más destacados entre las voces críticas, son muy diferentes en función del ámbito disciplinar y del grado con el que cada comunidad científica haya avanzado en la cultura de la autorregulación de los autores, en los mecanismos de supervisión y moderación por parte de los gestores de los repositorios, y en el ejercicio espontáneo y participativo del open peer review sobre los documentos depositados.

En cualquier caso, el preprint no se entiende en la actualidad al margen de su depósito en un repositorio: aunque cualquier manuscrito difundido por ejemplo en la página web personal de un autor podría tener características similares, su presentación aislada, sin las funcionalidades de un repositorio que permiten la supervisión, trazabilidad, persistencia y descubribilidad, limita su validez como instrumento de la ciencia abierta. En este sentido, la elección de dónde se deposita y cómo se presenta el preprint son aspectos importantes a la hora de maximizar ventajas y minimizar inconvenientes, y para ello es conveniente tener bien presentes las diversas recomendaciones y ejemplos de buenas prácticas (Tidjink et al., 2020; Beck et al., 2020). En ellas se apela a la claridad y transparencia en los procedimientos de carga y visualización de los preprints en los repositorios (indicando siempre la versión del documento, enlazando sus diversas ocurrencias, etc.) y también a la responsabilidad y formación de los autores (teniendo siempre presentes las limitaciones y provisionalidad de los preprints).

Todo proceso de transformación en el ámbito de la comunicación social tiene un impacto en personas y grupos de interés. Por ello, la evolución de la penetración del sistema de preprints dependerá de la posición que al respecto tengan los diversos protagonistas de la cadena de comunicación científica en cada ámbito del conocimiento (autores, lectores, editores, evaluadores, etc.), con grandes diferencias entre las ciencias experimentales, las sociales y las humanidades (Laporte, 2017). Por ello, son muy necesarios los estudios dedicados a captar la opinión y el comportamiento que al respecto tienen los posibles actores del sistema. Sarabipour et al. (2019) presentan la valoración de un grupo de investigadores jóvenes sobre las oportunidades y los riesgos de los preprints en la comunicación científica. Por su parte, en un informe de ASAPbio (Funk et al., 2020) se presentan los resultados preliminares de una encuesta internacional cumplimentada por 
546 personas vinculadas a la comunicación científica (la mayoría de ellos, investigadores): el cuestionario utilizado solicitaba información sobre su experiencia en preprints (como autor, lector, revisor, etc.) y una valoración sobre los diversos beneficios y preocupaciones respecto a su uso. Chiarelli et al. (2019) captaron mediante entrevistas la opinión de investigadores, administradores de repositorios y representantes de organismos financiadores de la investigación y de agencias de evaluación, y elaboraron un estudio de prospectiva en el que plantean un buen número de sombras sobre la consolidación de los preprints más allá de los ámbitos de ciencias duras (física, matemáticas, etc.) donde han devenido una parte estructural del sistema de comunicación científica. Todos esos incipientes estudios, apuntan claramente a la necesidad de tomar en consideración las diversas culturas de publicación de cada disciplina.

\section{Los congresos científicos ante su transformación digital}

En los últimos años se han abierto paso denominaciones como unconferences (Budd et al., 2015), amplified conferences (Guy, 2011), flipped conferences (Thomson, 2014), o non-real time web conferences (Arnal et al., 2020) entre otras, términos que tienen como denominador común la dinamización digital de las interacciones entre los participantes de reuniones científicas, con el objetivo de conseguir el aprovechamiento del valor añadido realmente genuino que representa reunir un grupo de personas en un mismo lugar al mismo tiempo, o directamente conseguir un efecto similar de forma virtual (Mulgan, 2015). La canalización anticipada y vía preprints de las comunicaciones como forma de preparar la celebración de un congreso abre la puerta a focalizar energías en la interacción entre participantes, por lo que los preprints se pueden contemplar como un componente más del debate sobre cómo han de ser los congresos científicos en el futuro más inmediato. Como apunta Sohn (2018), a los asistentes se les deberá ofrecer un protagonismo creativo en el desarrollo de este tipo de eventos, una tarea de la cual ser partícipe, especialmente a los inscritos que no contribuyen con ninguna comunicación. El debate público sobre los preprints vinculados a cada sesión de un congreso, entendido como una vía de crowd-based peer review, sería una forma clara de participación.

El estudio que nos ocupa se centra en analizar la experiencia de convergencia entre preprints y la gestión de las comunicaciones en un congreso, un tema al que la bibliografía no parece haber dedicado atención. Se trata de un vacío en cierta medida sorprendente, ya que buena parte de los puntos fuertes de los preprints tienen que ver con algunas de las razones de ser que históricamente han llevado a la celebración de congresos. La consideración de las comunicaciones de congreso como una forma de publicación preliminar de investigaciones en curso comparte buena parte de las características de los preprints, y permite suponer que la transformación digital de los congresos puede tener una importante repercusión en el futuro de los conference proceedings como tipología 
documental. Por otra parte, la función de dinamización del debate científico propia de los congresos, junto a la facilitación del networking entre investigadores, constituye una palanca eficaz para desencadenar un espacio de open peer review.

Si bien no se ha localizado bibliografía que analice experiencias similares a la que tratamos en este trabajo, son numerosos los congresos que se limitan a dar publicidad de los resúmenes de las contribuciones y no publican actas completas, por lo que no piden a los autores las comunicaciones definitivas en formato camera ready, ni realizan la exigente tarea de publicar las actas tras la revisión y edición de los textos por parte de la organización. Como alternativa a las actas, es frecuente que se disponga de acuerdos con revistas que publican números monográficos dedicados a congresos relacionados con su alcance temático, o que admiten manuscritos procedentes de comunicaciones de congresos en su flujo normal de publicación. En ambos casos, la conversión de las comunicaciones en artículos de revista no es automática y por ello podemos encontrar en la bibliografía ejemplos que analizan la tasa de conversión de las comunicaciones de congresos en artículos, especialmente numerosos en áreas como la medicina en las que el registro del conocimiento científico y la evaluación de la investigación se sustentan en las revistas y los congresos son un paso preliminar en la comunicación de resultados (p.e. Chen et al., 2020; Javidan et al., 2019; Scherer et al., 2018). Se trata de estudios de una naturaleza similar a los que analizan la conversión de preprints en artículos formalmente publicados (p.e. Lin et al., 2020). En cualquier caso, se puede considerar que hay un potencial para considerar los congresos y los preprints como realidades conectadas que pueden retroalimentarse.

\section{Métodos}

Con el propósito de captar el comportamiento y las percepciones de los autores de comunicaciones sobre el modelo de congreso desarrollado durante el IX Encuentro Ibérico EDICIC, se envió un formulario de encuesta en línea gestionado con Microsoft Forms (ver enlace en Anexo) a los 102 autores de correspondencia responsables de las 113 comunicaciones que finalmente se presentaron oralmente. Dado que el estudio pretendía evaluar el funcionamiento y los resultados del sistema de presentación de las comunicaciones mediante preprints y sin la posterior edición de las actas del encuentro, la encuesta no se envió a autores de pósteres, participantes en el consorcio doctoral, ni a los asistentes en general. Se optó por enviar el formulario únicamente a los autores de correspondencia de las comunicaciones, considerando que el liderazgo asumido en dicha función respecto del resto de coautores permitía obtener una información única y representativa de lo sucedido en cada una de las comunicaciones, sin duplicaciones e inconsistencias.

Para facilitar la participación, se ofrecieron dos versiones idénticas del cuestionario de encuesta, uno en portugués y el otro en español. El enlace al formulario en línea se envió 
nominalmente por correo electrónico el día 1 de mayo de 2019, se realizaron dos recordatorios a lo largo de dicho mes, y se obtuvo una tasa de respuesta del $65 \%$. Con dicho calendario se buscó conocer, en los diez primeros meses tras la fecha de celebración del encuentro, el grado de conversión de las comunicaciones en manuscritos efectivamente presentados para su publicación en revistas y el recorrido experimentado hasta su publicación en los casos de éxito. Dado el número limitado de autores encuestados y su vínculo con un congreso concreto, los resultados se analizaron con un enfoque cualitativo de interpretación como aportación al debate sobre los eventos del área de Información y Documentación.

\section{Resultados}

\subsection{Perfil de los autores}

Sesenta y seis autores de correspondencia contestaron el cuestionario, 53 (80\%) lo hicieron en la versión en portugués y 13 (20\%) en la versión en español, una distribución bastante proporcional al número de comunicaciones presentadas en cada lengua. En cuarenta y seis de esas respuestas se obtuvo información sobre el perfil del participante. La mayoría de ellos (28 participantes, 61\%) se identificó como personal académico, seguido por los estudiantes de doctorado $(8,17 \%)$ y por los profesionales $(6,13 \%)$, mientras que en cuatro respuestas (9\%) se marcó la opción "otros". En cuanto a los años de experiencia en su posición laboral en el momento de la encuesta, 13 participantes (28\%) contaban con una trayectoria de menos de cinco años, 17 (37\%) entre cinco y quince años de experiencia y 16 (35\%) más de quince años.

\subsection{Experiencia previa en el uso de preprints}

Las tres primeras preguntas del cuestionario trataban sobre la experiencia con los preprints que tenían los participantes con anterioridad a la convocatoria del Encuentro EDICIC 2019 (Figura 1). En los resultados se pudo observar que el grado de familiaridad con esta tipología documental entre los participantes era más bien bajo en cuanto a lectura, y prácticamente insignificante a nivel de uso como vía de difusión de sus resultados de investigación.

En concreto, menos de la mitad de los encuestados (30 participantes, 45\%) declararon haber leído preprints de otros autores con anterioridad, mientras que 29 participantes (44\%) confirmaron que no tenían experiencia lectora de los mismos, destacando como expresión de la falta de familiaridad con esta tipología documental las 7 respuestas (10\%) en las que los participantes no estaban seguros de haberlos leído alguna vez. En cuanto a su uso como autores, tan solo 7 de ellos (11\%) tenían experiencia previa, algo de lo que carecían los restantes 59 participantes (89\%). Curiosamente, un porcentaje muy mayoritario (56 participantes, 85\%) manifestaron tener una percepción positiva sobre la fiabilidad y la calidad de los preprints del área de Información y Documentación, frente tan solo 4 participantes (6\%) cuyo juicio al respecto fue negativo, datos que contrastan con el bajo nivel de uso. 


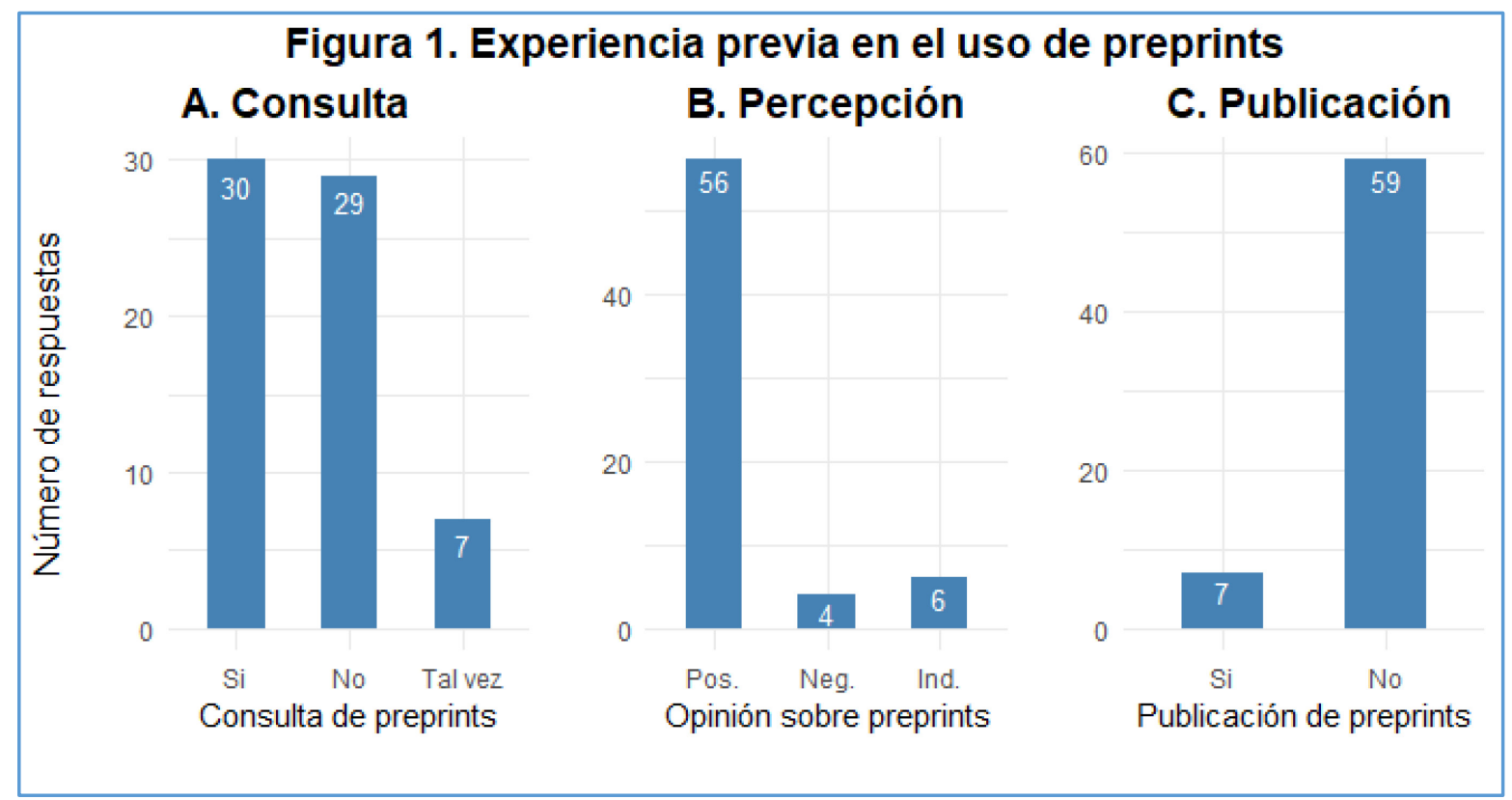

A los siete participantes que habían publicado un preprint con anterioridad a EDICIC 2019 (Figura 1c) se les preguntó mediante elección múltiple sobre las diversas motivaciones que les movieron a hacerlo en su día. Cinco participantes manifestaron que pretendieron "Fomentar el acceso abierto a la ciencia" y otros cinco "Dar visibilidad a los resultados obtenidos". En menor medida y con dos respuestas en todos los casos señalaron "Obtener retroacción y comentarios de colegas", "Acelerar la difusión de resultados de investigación evitando el retraso que implica la revisión, edición y publicación final en revistas científicas" y "Captar citas con una mayor inmediatez". En cuanto a su satisfacción en relación con la consecución de dichos objetivos, cinco participantes se manifestaron moderadamente satisfechos, mientras que uno se declaró altamente satisfecho y otro expresó indiferencia.

Pese al bajo número de participantes que declararon haber publicado preprints con anterioridad a EDICIC 2019, se observó una alta dispersión en la mención de los repositorios utilizados en respuesta a una pregunta de elección múltiple: E-LIS (tres participantes), OSF Preprints (dos), Zenodo (dos), repositorios institucionales (dos), arXiv.org (uno) y LISSA (uno).

\subsection{Depósito de la comunicación como preprint}

Contaron con preprint depositado un total de 86 comunicaciones de las 113 efectivamente presentadas durante el evento. En la distribución según los repositorios empleados (Tabla 1) destaca el dominio de E-LIS con un $55,81 \%$ de las comunicaciones que contaron con preprint. 
Tabla 1. Repositorios utilizados para depositar el preprint

\begin{tabular}{|l|c|c|}
\hline Repositorio & $\begin{array}{c}\text { número de } \\
\text { comunicaciones }\end{array}$ & \\
\hline E-LIS [http://eprints.rclis.org/] & 48 & $55,81 \%$ \\
\hline LISSA (LIS Scholarship Archive) [https://osf.io/preprints/lissa/] & 15 & $17,44 \%$ \\
\hline OSF Preprints [https://osf.io/preprints/] & 11 & $12,79 \%$ \\
\hline Repositorio Institucional (del propio centro del autor) & 5 & $5,81 \%$ \\
\hline arXiv.org [https://arxiv.org/] & 2 & $2,33 \%$ \\
\hline Dropbox [https://www.dropbox.com/] & 2 & $2,33 \%$ \\
\hline Zenodo [https://zenodo.org/] & 2 & $2,33 \%$ \\
\hline MDPI Preprints [https://www.preprints.org/] & 1 & $1,16 \%$ \\
\hline Total de comunicaciones depositadas & $\mathbf{8 6}$ & $\mathbf{7 6 , 1 1 \%}$ \\
\hline Total de comunicaciones NO depositadas & $\mathbf{2 7}$ & $\mathbf{2 3 , 8 9 \%}$ \\
\hline
\end{tabular}

Como complemento a estos datos registrales de lo sucedido, se consideró importante que la encuesta ayudara a determinar por qué, o por qué no, se realizó el depósito y las circunstancias que rodearon dicho proceso. Así, a la pregunta de si habían depositado como preprint su comunicación antes del inicio del encuentro, 48 participantes (73\%) respondieron afirmativamente, un porcentaje que se ajusta bastante al observado en los datos registrados por la organización para el total de comunicaciones. Los 18 restantes (27\%) justificaron las razones por las que no lo hicieron de la siguiente forma: en cinco casos por "Falta de tiempo", también en cinco por "Falta de reconocimiento de los preprints en los procesos de evaluación de la investigación", en tres casos por "Miedo a tener problemas para la posterior publicación en revistas que piden manuscritos originales no publicados" y en dos a causa de "Instrucciones poco claras por parte de la organización". A pesar de que el cuestionario ofrecía la opción, ninguno de los 18 participantes que no depositaron la comunicación como preprint justificó su decisión en proteger su creación intelectual y evitar ser plagiado.

A los participantes también se les preguntó si habían experimentado alguna dificultad al depositar su trabajo. Seis de ellos respondieron que habían tenido numerosas dificultades, mientras que 21 declararon algunas dificultades. En respuesta a una pregunta abierta, algunos participantes detallaron las dificultades, en ocasiones relacionadas con un cierto desconocimiento de los servidores de preprints adecuados:

Algumas plataformas não aceitavam preprints em português. Por não saber disso, tive dificuldade até encontrar a plataforma certa. Também tive algumas dificuldades para fazer a submissão, mas foram poucas.

Tive dificuldade em localizar uma ferramenta que possibilitasse a publicação do preprint. 
Encontrar a plataforma mais adequada para o efeito.

Dificuldade de localização do repositor.

Tive que submeter em dois servidores, pois o primeiro aceitava textos somente em inglês. Demorou mais ou menos uma semana após o prazo dado pelo evento de envio para que o segundo servidor publicasse o preprint.

En otros casos, los participantes experimentaron dificultades técnicas en su interacción con los mecanismos para subir el preprint a las plataformas:

A plataforma de preprint não é "friendly".

Não estava a conseguir disponibilizá-lo e torná-lo público. O link não abria.

Dificuldade em lidar com os procedimentos da plataforma, um pouco confusos.

Dificuldade, por falta de experiência, no processo que era necessário seguir.

Desconhecimento dos procedimentos e softwares envolvidos.

As especificações não são claras.

A plataforma foi difícil de entender, o prazo para colocar no ar muito longo, com relação aos direitos autorais.

Problemas técnicos con la plataforma (identificación no válida...) sin más importancia.

\subsection{Percepciones sobre el uso de preprints en la celebración de EDICIC 2019}

Algunas preguntas del cuestionario se dedicaron a captar el grado de acuerdo o desacuerdo con las innovaciones introducidas en EDICIC 2019 en relación al uso de preprints. En dos de ellas se solicitó la opinión sobre en qué medida ayudó a un mejor desarrollo del encuentro la disponibilidad anticipada de los preprints con el texto completo de las comunicaciones y la reducción del tiempo de presentación de las comunicaciones para dedicar espacio a los debates entre los autores y los asistentes.

La mayoría de los encuestados manifestaron estar de acuerdo con ambas innovaciones (Figuras $2 \mathrm{~A}$ y $2 \mathrm{~B}$ ). Así, 47 participantes (71\%) manifestaron su acuerdo, ya fuera total (23 participantes, 35\%), o parcial (24 participantes, 36\%), con la idea de que un congreso funciona mejor cuando se han puesto previamente los preprints a disposición de los 
asistentes. De forma similar, 39 participantes (85\% de los 46 que respondieron esta pregunta) manifestaron su acuerdo en limitar el tiempo de presentación por parte de los autores comunicaciones para dar más importancia al debate con los asistentes, 26 de los cuales $(57 \%)$ se manifestaron totalmente de acuerdo y $13(28 \%)$ parcialmente.

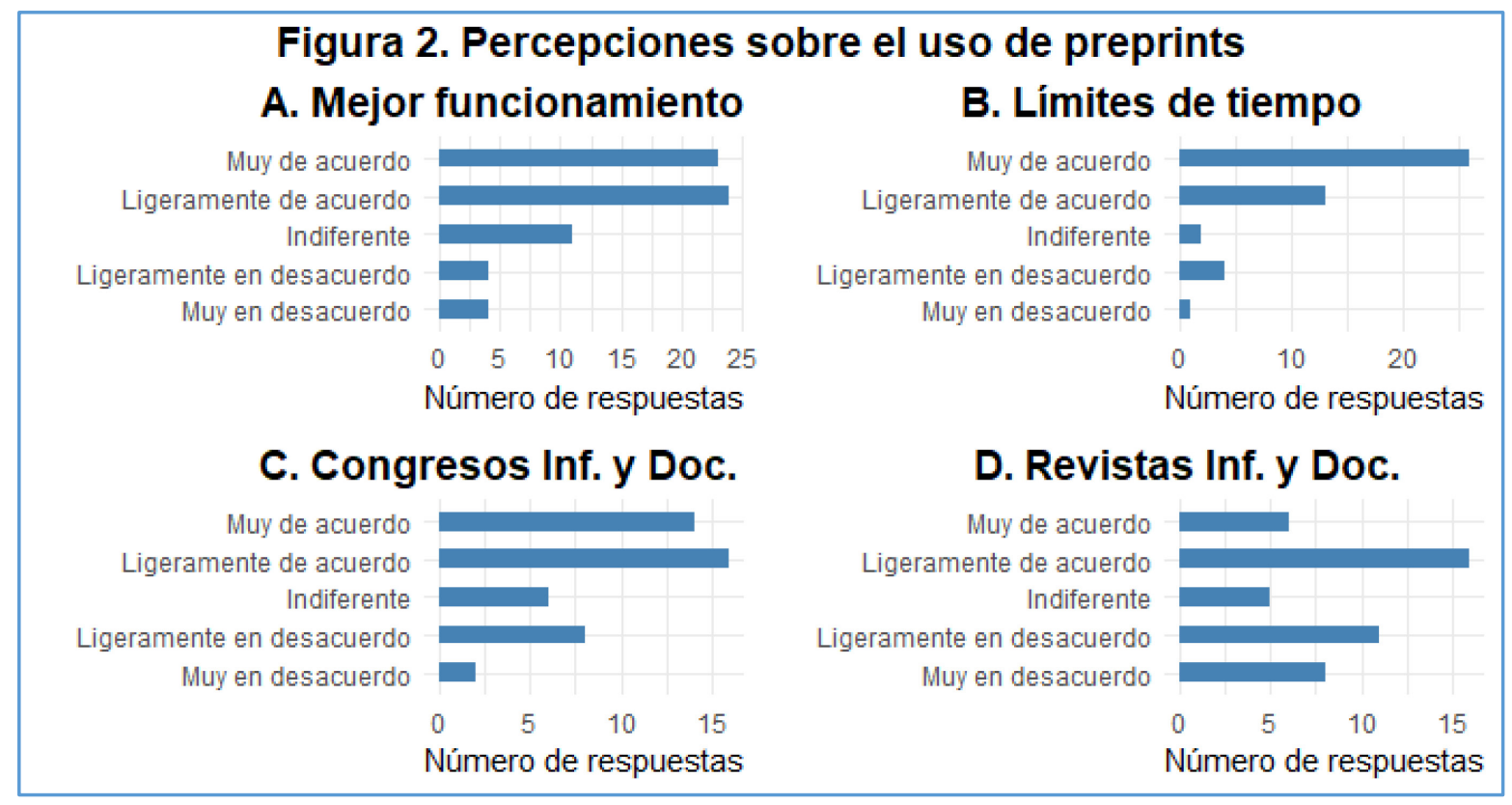

También se preguntó a los participantes si verían con buenos ojos que el modelo usado en EDICIC 2019 se aplicara en otros congresos del área de Información y Documentación, y si pensaban que las revistas del área estaban preparadas para este nuevo modelo de comunicación científica alineado con los principios de la ciencia abierta (Figuras 2C y 2D). En relación con el tema de la extensión del modelo a otros congresos del área, 30 participantes (65\% de los 46 que respondieron la pregunta) manifestaron su acuerdo, bien total (14 participantes, 30\%), bien parcial (16 participantes, 35\%), con la generalización del modelo aplicado en EDICIC 2019. Sin embargo, diez participantes (22\%) manifestaron su desacuerdo con dicha idea, ocho de ellos parcialmente (17\%) y dos totalmente (5\%). En lo referente a si las revistas del área estaban preparadas para la incorporación de los preprints en el marco de los principios de la ciencia abierta se pudo observar mayor controversia: 22 participantes ( $48 \%$ de los 46 que respondieron) pensaban que las revistas estaban preparadas, pero no así 19 de ellos (41\%); 5 de ellos no se manifestaron de acuerdo ni en desacuerdo. 


\subsection{Publicación formal de los preprints como artículos de revista}

Cada autor de correspondencia podía enviar un máximo de dos contribuciones al encuentro. Siete de los participantes así lo hicieron, por lo que se contabilizaron un total de 73 comunicaciones correspondientes a los 66 autores que respondieron la encuesta, en la que se les preguntó por el estado de publicación de su comunicación como artículo de revista, en caso de que hubieran ejercido dicha opción.

En el momento de realización de la encuesta (mayo de 2020), cuando aproximadamente habían pasado 10 meses desde la celebración del Encuentro EDICIC 2019, sobre un total de 73 comunicaciones representadas en las 66 respuestas al cuestionario, 34 (47\%) habían sido enviadas a alguna revista para su publicación formal. Sólo en 26 casos los autores indicaron el título de la revista elegida (Tabla 2), todos ellos títulos en portugués o en español y cuyos editores habían dado su conformidad a recibir preprints de EDICIC. Un autor canalizó el preprint como capítulo de libro.

Tabla 2. Lista de las revistas a las que los participantes enviaron sus manuscritos

\begin{tabular}{|l|c|}
\hline Revista & Envíos \\
\hline Informação \& informação & 6 \\
\hline Revista Ibero-americana de Ciência da Informação & 6 \\
\hline Biblios: Journal of Librarianship and Information Science & 5 \\
\hline Anales de Documentación & 2 \\
\hline Hipertext.net & 2 \\
\hline Ciência da Informação & 1 \\
\hline El Profesional de la Información & 1 \\
\hline Ibersid: revista de sistemas de información y documentación & 1 \\
\hline Páginas a\&b - arquivos e bibliotecas & 1 \\
\hline Revista Bibliomar & 1 \\
\hline Revista Cubana de Información en Ciencias de la Salud & 1 \\
\hline [Cap. libro] & 1 \\
\hline
\end{tabular}

Las respuestas a este apartado permiten constatar que los autores que habían depositado como preprint su comunicación antes del encuentro tuvieron una mayor inclinación a enviar posteriormente su trabajo a una revista para su publicación formal como artículo. La Figura $3 \mathrm{~A}$ muestra que postularon su trabajo ante una revista 26 (54\%) de los 48 autores que depositaron el preprint; por el contrario, entre los 18 que no depositaron la comunicación como preprint tan solo 5 (28\%) enviaron su trabajo a una revista. 


\section{A. Preprints}

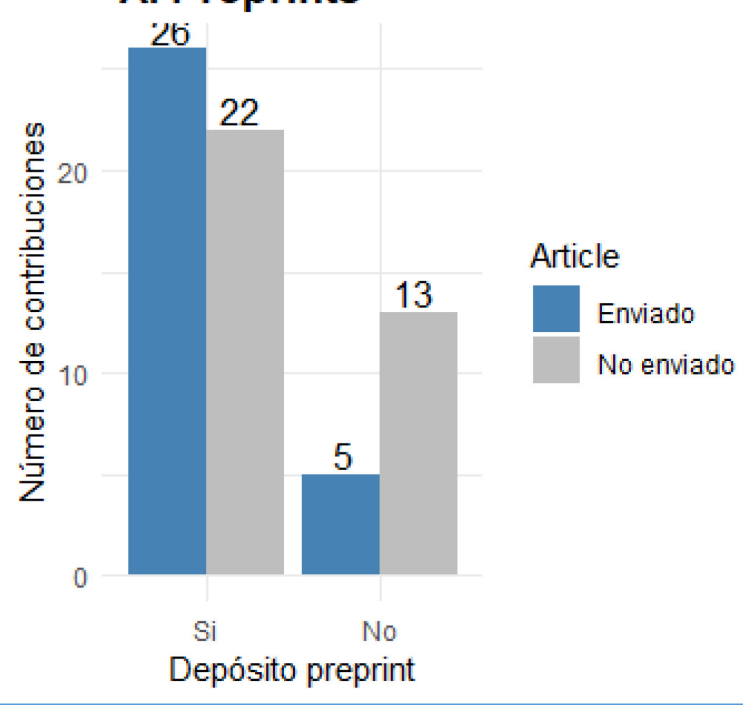

Figura 3. Preprints y artículos

\section{B. Artículos}

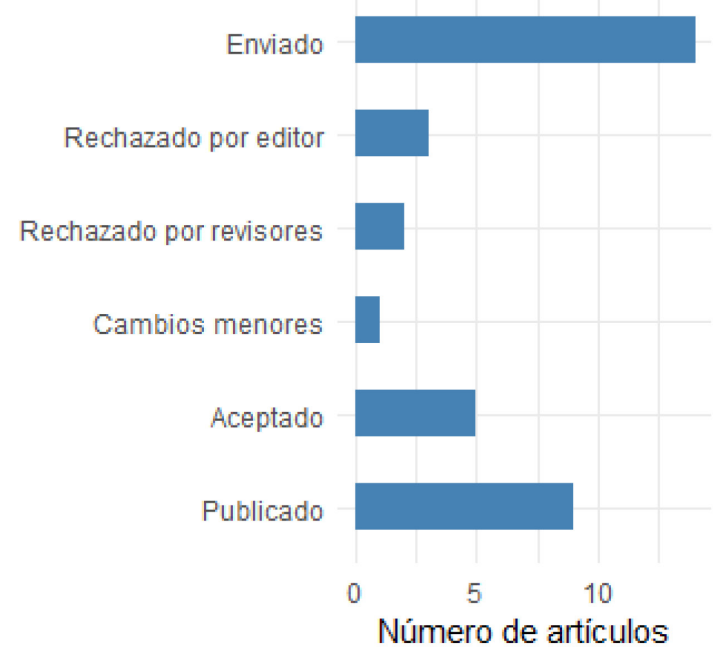

Ahora bien, tan solo 15 trabajos de 34 enviados habían sido ya aceptados: nueve habían sido ya publicados, cinco estaban a la espera de publicación y uno estaba pendiente de cambios menores. Entre los 19 envíos restantes que no contaban con veredicto de aceptación, cinco correspondían a manuscritos rechazados a nivel del filtro editorial inicial o de la revisión por pares, mientras que para 14 de ellos las revistas habían acusado recibo del envío, pero no habían emitido decisión alguna (Figura 3B).

También se preguntó a los participantes si habían introducido mejoras en el manuscrito a partir de la retroalimentación recibida durante la celebración del encuentro, o durante los meses de circulación del preprint, antes de postular el trabajo ante alguna revista. Desafortunadamente, solo se obtuvieron cuatro respuestas a esta pregunta y únicamente en dos casos se apuntó el resultado de esas interacciones como vía de mejora.

\subsection{Comentarios captados en la pregunta abierta final}

Una última pregunta abierta requería que los participantes proporcionaran los comentarios que considerasen oportunos sobre el desarrollo del encuentro y el uso de preprints. Dieciséis participantes aprovecharon esta oportunidad y realizaron comentarios diversos, que al margen de felicitaciones por el hecho de intentar innovar en el modelo de encuentro y por la realización de la encuesta, se pueden resumir en los siguientes puntos:

a) Preocupación por la falta de reconocimiento de su participación en el encuentro EDICIC Ibérico al no publicarse actas del encuentro. Temían que los preprints no tuvieran el mismo nivel de reconocimiento que las comunicaciones publicadas dentro de unas actas bien editadas bajo el sello editorial de los organizadores, y como parte de una serie de encuentros anteriores con actas reconocidas: 
Penso ser o EDICIC um congresso muito importante, mas que, ao meu ver, não valoriza dessa forma os trabalhos apresentados. É a minha percepção ao submeter o trabalho e apresentá-lo.

O evento EDICIC é muito bom e respeitado pelos pesquisadores. Contudo, se o evento continuar a não fazer publicações, corre o risco de perder participantes. O EDICIC é um evento caro para os estudantes de mestrado e doutorado, por isso, deveria oferecer um bom nível de publicação para os participantes.

Nas atuais regras de avaliação de desempenho docente, o facto de haver um livro de atas que está finalizado no momento do congresso ou posteriormente, dá-nos mais segurança quanto à produção de um manuscrito que, tendo passado pelos canais habituais de publicação (arbitragem científica a cargo de revisores), será efetivamente publicado.

Gosto muito do EDICIC. Considero esse evento de extrema importância para a área e só parabenizo pela melhoria quem vem ocorrendo nos últimos anos.

b) Doble esfuerzo al tener que pasar dos evaluaciones, la de los organizadores del encuentro y posteriormente la de la revista. La alternativa de enviar el preprint para su publicación posterior en una revista se experimentó por parte de algunos participantes como un esfuerzo adicional. El riesgo de pérdida de inscripciones se apuntó como una amenaza para este tipo de congresos en caso que los investigadores acaben valorando como menos costoso ir directamente a las revistas, sin pasar por congresos en los que se use el modelo de preprints para las comunicaciones:

O preprint não conta como publicação científica e os autores precisam modificar o trabalho para submeter em uma revista. É um trabalho duplo: primeiro evento e depois revista. O estudante gasta mais tempo e dinheiro com esse processo, assim os alunos podem preferir submeter para uma revista direto, porque reduz o tempo e o gastos.

Senti-me a realizar duas tarefas (o que é perturbador para o investigador) - 1) submeter para o congresso; 2) submeter para a revista - e senti que podia falhar nalguma delas. Enfim, o nosso investimento é alto e o sistema de recompensa pode não acompanhar as nossas expetativas, em caso de congressos em que os livros de ata não são publicados (o preprint é publicado, mas o artigo pode ser ou não - o autor não se sente, neste caso, recompensado - sente-se, antes, "punido". Há que pensar numa forma de garantir que ambas as situações existam (coexistência do preprint e do livro de atas). 
c) El vínculo y el compromiso de las revistas con el encuentro debería ser más explícito. Así, algunos participantes sugirieron que, en caso de descartarse las actas formalmente publicadas, los organizadores de la conferencia deberían llegar a acuerdos con los editores de revistas para facilitar la publicación de las comunicaciones como artículos. Algunos comentarios pusieron de manifiesto que los ritmos de recepción, designación de evaluadores, evaluación y respuesta final por parte de algunas revistas son manifiestamente mejorables:

A dificuldade de publicar consiste na demora das revistas e também não é toda revista que aceita o preprint. Minha sugestão é que o EDICIC faça parcerias com essas revistas e publique um número especial com os artigos aceitos no evento. $A$ publicação é muito importante para o pesquisador. Ao deixar a publicação apenas como preprint, o evento EDICIC corre o risco de ter menos pesquisadores interessados em participar do evento.

Por fim, submeti o artigo, tanto no repositório de preprint, quanto no período cientifico $X X X X X$, constante na lista de revistas alinhas em parceria com evento. Entretanto, desde 08/06/2019, dia da submissão, que o artigo mantém-se em aguardando designação. Talvez, tenha faltado um maior acerto entre o evento e a revista. [nota de transcripción: la encuesta se aplicó durante mayo de 2020]

Minha sugestão é que os organizadores combinem com editores de boas revistas a publicação dos trabalhos.

Outro fator é que o evento sugeriu algumas revistas em que os artigos poderiam posteriormente ser publicados e no entanto, não foi o que ocorreu: meu segundo trabalho está há quase um ano em avaliação na revista XXXXX, sem qualquer avanço no processo de análise. Isso também é muito prejudicial, uma vez que ir até o evento é um investimento por parte do investigador - financeiro e de tempo de pesquisa ao desenvolver o trabalho e portanto, espera-se que esse esforço seja recompensado por meio da publicação.

d) Ruptura con una tradición que permite una panorámica de la dinámica académica del área en Iberoamérica. Algunos encuestados se quejaron de que la ausencia de actas de estos encuentros impide consultar todas las contribuciones en un solo lugar y dificulta la realización de futuros estudios sobre las características y evolución del área:

Concordo com a sistemática dos preprints, mas gostava de ver a publicação em atas, onde encontraríamos todos os trabalhos apresentados.

Achei super válida a utilização de preprints e a disponibilização dos trabalhos previamente às apresentações. Minha única ressalva foi a não publicação de anais dos eventos, discordo veementemente dessa prática. Os anais muitas vezes são 
utilizados como objeto de pesquisa de novas investigações (como foi o caso de um dos trabalhos que eu apresentei no evento) e não reunir os trabalhos em um único documento, é extramente prejudicial pra esse tipo de pesquisa.

e) La cultura de los preprints y de la ciencia abierta todavía no está consolidada entre algunas revistas del área de Información y Documentación. Según la experiencia de algunos participantes, algunos editores de revistas del área de Información y Documentación son reacios a publicar como artículos aquellas contribuciones que han sido previamente archivadas en repositorios de preprints, y eso cuestiona el futuro de la propuesta de innovación con la que se experimentó durante EDICIC 2019:

Penso que os editores de revistas não estão muito bem preparados. A mim, aconteceu-me de me solicitarem a eliminação imediata do preprint [...], porque consideraram, indevidamente, que eu estava a tentar publicar um artigo que já havia sido publicado (porque encontraram o preprint na Internet e isso deduziram). Tive de explicar que não era o caso.

f) El incremento de la interacción y el debate en las sesiones de comunicaciones, objetivo último que se pretendía con la difusión anticipada de las comunicaciones, no se alcanzó plenamente. Si bien un buen número de participantes manifestaron su acuerdo en las preguntas cerradas de la encuesta con el modelo empleado para la difusión anticipada del texto completo de las comunicaciones, en este espacio de comentarios se registraron interesantes observaciones sobre los problemas de tiempo y de falta de compromiso en la lectura anticipada de comunicaciones entre los asistentes. También se apuntó a una asignación insuficiente de tiempo para el desarrollo de las sesiones, que limitaron la profundidad del debate y de la interacción entre participantes y asistentes:

Gostei da iniciativa da pesquisa. Acredito em novos modelos de difusão da produção científica em meio digital. A produção dos preprints oriundos de instituições científicas validam os conteúdos de acesso aberto, entretanto, livros digitais autopublicados ainda tem sua reputação questionada. Precisamos de mecanismos que validem esse tipo de publicação.

Creo que funcionó mejor la publicación preprint para poder dejar las comunicaciones accesibles que para poder leerlas previamente. El debate, creo, surge de la presentación personal y de la posibilidad de comentarlo con los autores en ese mismo momento. Creo que la presentación personal tiene un valor difícilmente substituible.

Primeiramente, eu gostaria de parabenizar aos envolvidos pela iniciativa. Trata-se de uma prática necessária, como forma de avaliar e repensar, mas que não acontece com frequência nos eventos da área. [...] As discussões foram ricas, especialmente 
pelas pré-leituras, contudo, o pouco tempo de apresentação, em alguns momentos, não permitiu maior enfase dos palestrantes.

g) La prueba piloto del sistema de preprints, vista desde la perspectiva de los primeros meses de pandemia de Covid-19 durante el año 2020, dio pie a sugerencias en relación a aspectos organizativos y económicos:

Essa crise de coronavírus irá aumentar as dificuldades financeiras dos estudantes, por isso, o evento precisa dar o retorno da publicação. As discussões e avaliações dos trabalhos são excelentes. Os avaliadores fazem avaliação ética, construtiva e com muito respeito. Se os editores do EDICIC pensam que a publicação do preprint é pertinente, então sugiro permitir a participação online de estudantes para que eles tenham menos gastos.

\section{Discusión y conclusiones}

La consolidación de los preprints como canal para la rápida diseminación de los resultados de la investigación comporta cambios importantes en la comunicación científica, que afectan directamente a la edición de revistas académicas y al funcionamiento de los congresos. Ahora bien, en el área de Información y Documentación la penetración de esta tipología documental es débil, por lo que la experiencia desarrollada en EDICIC 2019 tuvo un valor experimental e iniciático importante para muchos asistentes, dado que el número de comunicaciones que contaron con preprint fue muy elevado, pese a que para muchos era su primera experiencia con ese formato.

El estudio ha permitido poner de manifiesto algunas de las principales barreras en el uso de preprints. En primer lugar, se ha constatado un escaso conocimiento del funcionamiento de los preprints entre los autores. Pocos encuestados habían consultado preprints y un número aún inferior los había publicado con anterioridad a su participación en el congreso. La diversidad de plataformas disponibles no parece contribuir a la consolidación de este formato documental. Entre los participantes que depositaron el preprint de su comunicación, los principales obstáculos encontrados hacían referencia a la dificultad para identificar una plataforma adecuada y, una vez localizada, a problemas técnicos resultado de la necesidad de familiarizarse con servicios que, aunque similares, presentan funcionalidades diversas.

El dominio aplastante de E-LIS [http://eprints.rclis.org/] entre los repositorios utilizados por los participantes en EDICIC 2019 hace pensar en la importancia que otorgaron los autores a una plataforma que les era más familiar, ya fuera como lectores o como autores. Se ha de tener en cuenta que el ámbito iberoamericano genera un volumen importante de los contenidos de E-LIS, aunque correspondan principalmente a informes técnicos, versiones 
postprint o publicadas de artículos de revista, versiones publicadas de comunicaciones, presentaciones ppt, o posters.

En segundo lugar, la información suministrada por los autores pone de relieve la percepción de un elevado grado de desconfianza entre los editores de revistas hacia los preprints. Como se pudo comprobar en la extensa ronda de contactos con revistas iberoamericanas del área de Información y Documentación para su inclusión en la página web del Encuentro certificando la aceptación de preprints, ciertamente algunas revistas son reticentes a publicar artículos que se han difundido previamente como preprints, y la mayoría no explicitan en su web la posición al respecto. Algunos editores no consideran el preprint como la versión previa del manuscrito sino como un documento distinto $y$, atendiendo al imperativo de publicar trabajos originales e inéditos, exigen que no exista coincidencia entre ambos documentos. Esto genera confusión entre los autores que temen ver disminuir las oportunidades de publicación de sus trabajos si se depositan como preprint. En el caso concreto del congreso EDICIC, algún autor ha tenido que recordar al editor su compromiso con el congreso.

Los resultados del estudio muestran que el esfuerzo de preparación de un preprint no es baldío, sino que predispone a favor de la posterior publicación en una revista, con un porcentaje superior de manuscritos publicados entre los autores que habían depositado el preprint de su comunicación. Ahora bien, el porcentaje de comunicaciones que finalmente se postularon como artículos de revista al cabo de diez meses no fue el esperado, siendo realmente muy pocos los artículos ya publicados en la fecha de realización de la encuesta.

Con el trabajo también se pretendía recoger la opinión de los encuestados sobre el modelo experimental desarrollado durante el encuentro. Si bien la mayor parte de los encuestados se muestra favorable a la iniciativa, los resultados alcanzados en términos de incremento de la interacción y del debate entre los asistentes fueron escasos. Más allá de la publicación de los preprints, el éxito del modelo requiere del compromiso de los participantes con la lectura previa de los trabajos puestos a su disposición por los autores y de una transformación en la dinámica de las sesiones que reduzca los tiempos de exposición en beneficio del debate. Ambos cambios necesitan un proceso de maduración que no será inmediato. Además, la sostenibilidad económica de muchos congresos descansa en el pago de las inscripciones por parte de las personas que presentan comunicaciones, por lo que otorgar el tiempo necesario a los debates implicaría aceptar menos comunicaciones, o alargar la duración del evento.

Por otro lado, los preprints no suplen el cierto reconocimiento que todavía tienen las actas de congreso. Si bien el valor de las mismas en procesos de evaluación y acreditación es escaso, el de los preprints es aún inferior. Además, en las últimas ediciones de los encuentros del Capítulo Ibérico de EDICIC se puede observar una creciente proporción de autores portugueses y brasileños frente a una disminución de los españoles, algo que puede tener relación con la diferencia de valor que agencias y universidades otorgan en cada caso. 
En consecuencia, la eliminación de las actas, unida a los elevados costes de desplazamiento e inscripción, pueden suponer un grave problema a muchas convocatorias de reuniones científicas de nuestra área. Si no hay actas formalmente publicadas, muchos investigadores pueden estar tentados de obviar la presentación de una comunicación en un congreso para postularse directamente en una revista.

En definitiva, debe tenerse en cuenta que el comportamiento de los investigadores está altamente condicionado por los criterios aplicados en los procesos de evaluación a los que son sometidos de manera periódica. Será difícil, por tanto, conseguir el compromiso de los autores con la publicación de preprints $-y$, en general, con las actividades que desea promover la ciencia abierta como la compartición de datos primarios, la publicación en acceso abierto o la apertura de los procesos de revisión- si la adopción de estos comportamientos no tiene un correlato en los criterios aplicados por las agencias de evaluación y acreditación.

\section{Referencias}

Arnal, A., Epifanio, I., Gregori, P., \& Martínez, V. (2020). Ten simple rules for organizing a non-real-time web conference. PLOS Computational Biology, 16(3), e1007667. https://doi.org/10.1371/journal.pcbi.1007667

Balaji, B. P., \& Dhanamjaya, M. (2019). Preprints in scholarly communication: Re-imagining metrics and infrastructures. Publications, 7(1), art. no. 6, https://doi.org/10.3390/publications7010006

Beck, J., Ferguson, C. A., Funk, K., Hanson, B., Harrison, M., Ide-Smith, M., Lammey, R., Levchenko, M., Mendonça, A., Parkin, M., Penfold, N. C., Pfeiffer, N., Polka, J. K., Puebla, I., Rieger, O. Y., Rittman, M., Sever, R. \& Swaminathan, S. (2020). Building Trust in Preprints: Recommendations for Servers and Other Stakeholders. OSF Preprints. https://doi.org/10.31219/osf.io/8dn4w

Berg, J. M., Bhalla, N., Bourne, P. E., Chalfie, M., Drubin, D. G., Fraser, J. S., Greider, C. W., Hendricks, M., Jones, C., Kiley, R., King, S., Kirschner, M. W., Krumholz, H. M., Lehmann, R., Leptin, M., Pulverer, B., Rosenzweig, B., Spiro, J. E., Stebbins, M., ... Wolberger, C. (2016). Preprints for the life sciences. Science, 352(6288), 899 LP 901. https://doi.org/10.1126/science.aaf9133

Bourne, P. E., Polka, J. K., Vale, R. D., \& Kiley, R. (2017). Ten simple rules to consider regarding preprint submission. PLoS Computational Biology, 13(5), e1005473. https://doi.org/10.1371/journal.pcbi.1005473 
Brown, C. (2001). The E-volution of preprints in the scholarly communication of physicists and astronomers. Journal of the American Society for Information Science and Technology, 52(3), 187-200. http://doi.org/10.1002/10974571(2000)9999:9999\%3C::AID-ASI1586\%3E3.0.CO;2-D

Brown, C. (2003). The role of electronic preprints in chemical communication: Analysis of citation, usage, and acceptance in the journal literature. Journal of the American Society for Information Science and Technology, 54(5), 362-371. https://doi.org/10.1002/asi.10223

Budd, A., Dinkel, H., Corpas, M., Fuller, J. C., Rubinat, L., Devos, D. P., Khoueiry, P. H., Förstner, K. U., Georgatos, F., Rowland, F., Sharan, M., Binder, J. X., Grace, T., Traphagen, K., Gristwood, A., \& Wood, N. T. (2015). Ten simple rules for organizing an unconference. PLOS Computational Biology, 11(1), e1003905. https://doi.org/10.1371/journal.pcbi.1003905

Chen, J., Cao, Y., Wang, M., Gan, X., Li, C., \& Yu, H. (2020). Analysis of conference abstracts of prosthodontic randomised-controlled trials presented at IADR general sessions (2002-2015): a cross-sectional study of the relationship between demographic characteristics, reporting quality and final publication. BMJ Open, 10(2), e034635. https://doi.org/10.1136/bmjopen-2019-034635

Chiarelli, A., Johnson, R., Pinfield, S., \& Richens, E. (2019). Preprints and scholarly communication: An exploratory qualitative study of adoption, practices, drivers and barriers [version 2]. F1000Research, 8(June). https://doi.org/10.12688/f1000research.19619.2

Clarke, M. (2020, 29 de abril). Scientific and scholarly meetings in the time of pandemic. The scholarly kitchen. htps://scholarlykitchen.sspnet.org/2020/04/29/scientificand-scholarly-meetings-in-the-time-of-pandemic/

Confrey, E. A. (1966). Information exchange groups to be discontinued. Science, 154(3751), 843-843. https://doi.org/10.1126/science.154.3751.843

Elmore, S. A. (2018). Preprints: What role do these have in communicating scientific results? Toxicologic Pathology, 46(4), 364-365. https://doi.org/10.1177/0192623318767322

Eysenbach, G. (2000). The impact of preprint servers and electronic publishing on biomedical research. Current Opinion in Immunology, 12(5), 499-503. https://doi.org/10.1016/S0952-7915(00)00127-8

Feldman, S., Lo, K., \& Ammar, W. (2018). Citation count analysis for papers with preprints. 7 p. ArXiv.org. http://arxiv.org/abs/1805.05238 
Fry, N.K., Marshal, H., \& Mellins-Cohen, T. (2019). In praise of preprints. Microbial Genomics, 5(4), art. no. 000259. https://doi.org/10.1099/mgen.0.000259

Fu, D.Y., \& Hughey, J.J. (2019). Releasing a preprint is associated with more attention and citations for the peerreviewed article." eLife, 8, e52646.

https://doi.org/10.7554/eLife.52646

Funk, K., Meadows, A., Mendonça, A., Rieger, O., Swaminathan, S. (2020, Julio 27).

Preprint authors optimistic about benefits: preliminary results from the \#bioPreprints2020 survey. ASAPbio. https://asapbio.org/biopreprints2020-surveyinitial-results

Ginsparg, P. (2011). ArXiv at 20. Nature 476, 145-147 (2011). https://doi.org/10.1038/476145a

Guy, M. (2011). 10 cheap and easy ways to amplify your event. Ariadne, 66. http://www.ariadne.ac.uk/issue/66/guy/

Henneken, E. A., Kurtz, M. J., Eichhorn, G., Accomazzi, A., Grant, C., Thompson, D., \& Murray, S. S. (2006). Effect of e-printing on citation rates in astronomy and physics. ArXiv.org. http://arxiv.org/abs/cs/0604061

Javidan, A. P., Naji, F., Ali, K. P., Rapanos, T., \& Harlock, J. (2019). Factors relating to the publication rate of abstracts presented from 2012 to 2015 at the Society for Vascular Surgery Annual Meeting. Journal of Vascular Surgery, 69(6), 1909-1917. https://doi.org/10.1016/j.jvs.2018.09.034

Laporte, S. (2017). Preprint for the humanities: fiction or a real possibility? Studia Historiae Scientiarum 16, 367-378. http://doi.org/10.4467/2543702XSHS.17.014.7715

Lin, J., Yu, Y., Zhou, Y., Zhou, Z., \& Shi, X. (2020). How many preprints have actually been printed and why: a case study of computer science preprints on arXiv. Scientometrics, 124(1), 555-574. https://doi.org/10.1007/s11192-020-03430-8

Mulgan, G. (2015). Meaningful meetings: how can meetings be made better? London: Nesta. https://media.nesta.org.uk/documents/meaningful_meetings.pdf

Neylon, C., Pattinson, D., Bilder, G., \& Lin J (2017). On the origin of nonequivalent states: How we can talk about preprints. F1000Research, 6, art. 608. https://doi.org/10.12688/f1000research.11408.1

Ng, W. (2017). Preprints as medium for communicating new ideas, hypotheses, data, analysis and beyond. PeerJ Preprints. https://doi.org/10.7287/peeri.preprints.3154v1 
Packer, A. L., Santos, S. \& Meneghini, R. (2017, Febrero 22). SciELO Preprints en camino. SciELO en Perspectiva. https://blog.scielo.org/es/2017/02/22/scielo-preprints-encamino/

PLOS (2021). Open Science. Preprints. https://plos.org/open-science/preprints/

Rodríguez, E.G. (2019). Preprints and preprint servers as academic communication tools. Revista Cubana de Información en Ciencias de la Salud, 30(1). http://www.acimed.sld.cu/index.php/acimed/article/view/1324/802

Sarabipour, S., Debat, H.J., Emmott, E., Burgess, S.J., Schwessinger, B., Hensel, Z. (2019). On the value of preprints: An early career researcher perspective. PLoS Biology, 17(2): e3000151. https://doi.org/10.1371/journal.pbio.3000151

Scherer, R. W., Meerpohl, J. J., Pfeifer, N., Schmucker, C., Schwarzer, G., \& von Elm, E. (2018). Full publication of results initially presented in abstracts. Cochrane Database of Systematic Reviews, Issue 11, Art. No. MR000005. https://doi.org/10.1002/14651858.MR000005.pub4

Seminario CRICC (2019). ¿Qué es esto de los preprints... y cómo me afecta? Vídeo del webinar disponible en YouTube: https://youtu.be/hGAlpsgP5W0

Sheldon, T. (2018). Preprints could promote confusion and distortion. Nature, 559, 445. https://doi.org/10.1038/d41586-018-05789-4

Sohn, E. (2018). The future of the scientific conference. Nature, 564(7736), S80-S82. https://doi.org/10.1038/d41586-018-07779-y

Teixeira da Silva, J. A., \& Dobránszki, J. (2019). Preprint policies among 14 academic publishers. Journal of Academic Librarianship, 45(2), 162-170. https://doi.org/10.1016/j.acalib.2019.02.009

Tennant, J., Gatto, L., \& Logan, C. (2018). Preprints: help not hinder journalism. Nature 560, 553. https://doi.org/10.1038/d41586-018-06055-3

Tennant, J., Bauin, S., James, S. \& Kant, J. (2018). The evolving preprint landscape: Introductory report for the knowledge exchange working group on preprints. MetaArXiv Preprints. https://doi.org/10.31222/osf.io/796tu

Thomson, P. (2014, 22 de septiembre). Conference blog- let's flip the format! Patter. https://patthomson.net/2014/09/22/conference-blog-flipping-the-format/

Tijdink, J., Malicki, M., Bouter, L. \& Gopalakrishna, G. (2020, 23 de septiembre). Are preprints a problem? 5 ways to improve the quality and credibility of preprints. $L S E$ Impact Blog. https://blogs.Ise.ac.uk/impactofsocialsciences/2020/09/23/arepreprints-a-problem-5-ways-to-improve-the-quality-and-credibility-of-preprints/ 
Wang, Z., Chen, Y., \& Glänzel, W. (2020). Preprints as accelerator of scholarly communication: An empirical analysis in Mathematics. Journal of Informetrics, 14(4), 101097. https://doi.org/10.1016/j.joi.2020.101097

Wang, Z., Glänzel, W., Chen, Y. (2020). The impact of preprints in Library and Information Science: An analysis of citations, usage and social attention indicators. Scientometrics, 125(2), 1403-1423. https://doi.org/10.1007/s11192-020-03612-4

\section{Anexos}

Formulario de encuesta disponible, con mención al consentimiento informado en: https://figshare.com/articles/dataset/FormularioEncuestaEDICIC2019 pdf/13564199

Declaración CRediT de las contribuciones de cada autor:

- Cristóbal Urbano: Conceptualización, Metodología, Análisis formal, Redacción borrador original, Redacción - revisión y edición.

- Sara Tafalla: Investigación, Curación de datos, Análisis formal, Redacción - revisión y edición.

- Ángel Borrego: Metodología, Análisis formal, Validación, Visualización, Redacción borrador original, Redacción - revisión y edición.

- Ernest Abadal: Análisis formal, Validación, Redacción - revisión y edición

Preprint: v1.0 (12/01/2021), bajo licencia internacional CC BY 4.0 